\title{
Students' understanding and application of the area under the curve concept in physics problems
}

\author{
Dong-Hai Nguyen and N. Sanjay Rebello \\ Department of Physics, Kansas State University, Manhattan, Kansas 66506, USA
}

(Received 30 January 2011; published 28 June 2011)

\begin{abstract}
This study investigates how students understand and apply the area under the curve concept and the integral-area relation in solving introductory physics problems. We interviewed 20 students in the first semester and 15 students from the same cohort in the second semester of a calculus-based physics course sequence on several problems involving the area under the curve concept. We found that only a few students could recognize that the concept of area under the curve was applicable in physics problems. Even when students could invoke the area under the curve concept, they did not necessarily understand the relationship between the process of accumulation and the area under a curve, so they failed to apply it to novel situations. We also found that when presented with several graphs, students had difficulty in selecting the graph such that the area under the graph corresponded to a given integral, although all of them could state that "the integral equaled the area under the curve." The findings in this study are consistent with those in previous mathematics education research and research in physics education on students' use of the area under the curve.
\end{abstract}

DOI: 10.1103/PhysRevSTPER.7.010112

PACS numbers: $01.50 . \mathrm{Zv}$

\section{INTRODUCTION}

Many physics problems involve using an integral to calculate physical quantities from other nonconstant quantities. In most of these problems, the algebraic expressions of the functions to be integrated are provided or can be determined from the problem statements, so the integrals can be computed algebraically. There are also a few problems in which the integrals must be evaluated graphically using areas under the curves of the integrands. Many research studies have investigated students' problem solving with algebraic computation of integrals [1-3]. However, there have not been many studies on how students use graphical methods to evaluate definite integrals in physics problems.

In this study, we investigated students' understanding and application of the concept of area under the curve to evaluate definite integrals in several physics problems. These problems were designed to investigate whether students were able to recognize and use the concept, whether they understood what quantity the area represented, and whether they could match a definite integral with an area under a curve.

In the next section, we review some of the studies in mathematics and physics education research on students' understanding of integration and the relationship between a definite integral and an area under a curve. In Sec. III, we describe the methodology of our research study. We present our findings in Sec. IV and discuss how these

Published by the American Physical Society under the terms of the Creative Commons Attribution 3.0 License. Further distribution of this work must maintain attribution to the author(s) and the published article's title, journal citation, and DOI. findings support and extend other studies in mathematics and physics education research in Sec. V. The limitations of our study and our future work built on this study will be discussed in Sec. VI.

\section{LITERATURE REVIEW}

There have been several studies in mathematics education research on students' understanding of integration and the relationship between the definite integral and the area under a curve. Orton [4] investigated students' understanding of integration, the errors students made when solving integration problems, and the relationship between a definite integral and area under a curve. He interviewed 110 British students aged 16 to 22 on several limits and integration tasks. Many of these tasks involved finding areas under curves using the Riemann sum method and calculating the limit of that Riemann sum. Some other tasks asked students to prove basic properties of integration (such as the integral of a sum was the sum of integrals) using area under the curve. Orton found that the majority of students did not perceive the integral as the limit of a Riemann sum and talked about such limit as an approximation, not as an exact answer, although they had no difficulty evaluating a given Riemann sum. Similar results were found by Artigue [5], who also investigated students' understanding of differentiation and integration. Artigue found that although most of the students could perform routine procedures for finding the area under a curve, rarely could they explain their procedures. Some students did not even realize why they were doing it.

Ferrini-Mundy and Graham [6] interviewed a group of six students in calculus to reveal students' understanding of basic concepts of calculus (e.g., function, limit, continuity, 
derivative, and integral) and the interrelationships among those concepts. They investigated in detail the performance of one student in the interviews. They found that this student, like many others in the study, "interpreted the integral as a signal to "do something." This student perceived the definite integral as "the area between the graph of the function and the x-axis," while thinking of the Riemann sum of the areas of the small rectangles under the curve as the "proof" for that fact.

There were also studies that investigated students' ability to provide an abstract definition of the definite integral and the concept image students had for it. A concept image is a cognitive structure in an individual's mind that is associated with a specific mathematical concept [7]. Bezuidenhout and Olivier [8] analyzed students' written tests and interviews to reveal students' concept image of the definite integral. Specifically, they looked at students' process conception and object conception, which were two components that formed parts of the concept image that an individual had of the concept. They found that students inappropriately applied the limit law that "the limit of a sum is the sum of the limits" to the Riemann sum, which revealed their inappropriate process conception of the limit concept. Students also associated the definite integral with the area between the curve and the horizontal axis, which they perceived as being always positive. Therefore, they took the absolute values of the results of the integrals to obtain positive results. This erroneous "area conception" of integral was an example of the unsatisfactory conceptions which might be due to insufficient abstraction of the concept images of the integral. In our study, we also revealed students' misconception about the integral-area relation when they attempted to select the graph from among several graphs that corresponded to a predetermined integral representing a physical quantity.

Rasslan and Tall [9] also investigated the definition and images of the definite integral held by high school students in the United Kingdom. They found that "the majority do not write meaningfully about the definition of definite integral, and have difficulty interpreting problems calculating areas and definite integrals in wider contexts." They suggested strategies for teaching the definite integral concept. The strategy was to introduce the concept as "cases [that] extended the students' previous experience" and let the students experience it in use through a variety of examples covering a wide contextual range. Also on the topic of students' definition and image of the definite integral is the work of Grundmeier et al. [10]. They surveyed 52 students leaving a calculus class that covered the theory and techniques of integration. The survey was designed to explore students' ability to define the definite integral in words and symbols, to interpret and represent an integral graphically, to evaluate integrals, and to recognize the use of integrals in the real world. They found that students' knowledge of the definition of the integral did not affect their ability to perform routine calculation on the integrals. They also found that students could "perform integration as a procedure with limited understanding that they are finding the area under the curve and that this area is being found as a limit of estimations for that area."

Mahir [11] investigated the conceptual and procedural knowledge of 62 students who had successfully completed a one-year calculus course. These students were asked to solve five calculus problems relating the concepts of integral, integral-area relation, integral as a sum of areas, and the fundamental theorem of calculus. The first two problems (1 and 2) could be solved using integral formulas and techniques, so these problems could evaluate students' procedural knowledge. The next two problems (3 and 4) could be solved by using either the integral-area relation or symbolic integral techniques. The last problem (problem 5) was more complicated and required students to combine many concepts, so it served to evaluate students' conceptual knowledge. Mahir found that the students in his study did not have satisfactory conceptual understanding of the concepts being tested. He also concluded that the students following the conceptual approach also performed satisfactorily on procedural calculations and had a higher success rate than the students following the procedural approach. He suggested that concept-based instruction might help to improve students' conceptual understanding in calculus. This suggestion was supported by the study of Chapell and Killpatrick [12] who found that "students exposed to the concept-based learning environment scored significantly higher than the students in procedural-based environment on assessment that measures conceptual understanding as well as procedural skills."

All of the research mentioned above indicates that students who had taken calculus courses did not have satisfactory conceptual understanding of the integral concept and the integral-area relation although they might be very fluent in performing symbolic integral techniques or in calculating the area under a curve. This lack in conceptual understanding of the integral and the integral-area relation will become a stumbling block when students attempt to apply the area under the curve concept in real world problems, such as physics problems. Building on these previous studies in mathematics education, our study focuses on exploring the difficulties that students who have successfully completed integral calculus courses encounter when applying the area under the curve concept in physics problems. Specifically, we investigate whether students in calculus-based physics courses could recognize the application of the area under the curve in physics problems, whether they understood what quantity the area under the curve represented, and whether they could match a definite integral with the corresponding area under the curve when provided with several curves.

Thompson and Silverman [13] pointed out that for students to perceive the area under a curve as representing a 
quantity other than area (e.g., velocity, work), it was important that students considered the quantity being accumulated as a sum of infinitesimal elements that were formed multiplicatively. Thompson and Silverman proposed the accumulation model in which integration meant accumulating the bits that were made of two multiplicative quantities. This model emphasized the two "layers" of integration: the multiplicative layer when the bits were formed and the accumulating layer when the bits were accumulated.

Sealey [14] also emphasized the importance of understanding the structure of the integral with the ability to apply the area under the curve in physics problems. She investigated students' problem solving on "real world problems" involving integration in a calculus class. The "real world problems" in this study were physics problems in which physical quantities were calculated using integration. She found that students might be proficient in dealing with area under a curve, but they might not be able to relate such an area to the structure of a Riemann sum. She concluded that the area under the curve method could be a powerful tool to evaluate a definite integral only when students understood the structure of the definite integral.

In our study, we found evidence of students' failure in interpreting the meaning of the area under the curve when they did not perceive it as a Riemann sum and did not understand the structure of the Riemann sum. The hints that we provided to help students to recognize the use of the area under the curve and to interpret its meaning in physics problems were based on the structure of the Riemann sum which was consistent with the suggestion of Thompson and Silverman and Sealey.

There have been a few studies in physics education research that focus on how students apply the area under a curve method in evaluating integrals in physics problems. McDermott et al. [15] investigated students' difficulties in connecting graphs and physics in the context of kinematics. They identified two categories of difficulty students had with graphs. First, students had difficulties in connecting graphs to physics concepts, including discriminating between the slope and height of a graph, interpreting changes in height and changes in slope, relating one type of graph to another, matching narrative information with relevant features of a graph, and interpreting the area under a graph. Second, students had difficulties in connecting graphs to the real world, including representing continuous motion by a continuous line, separating the shape of a graph from the path of the motion, representing a negative velocity on a " $v$ vs $t$ " graph, representing constant acceleration on a " $a$ vs $t$ " graph, and distinguishing among different types of motion graphs.

In a problem involving finding displacement from a graph of " $v$ vs $t$," students had to find the area under the curve by counting the number of squares bounded by the curve and the $v=0$ axis and then multiplying it by the displacement that each square represented. They found that most of the difficulties students had were directly related to their "inability to visualize the motion depicted by the velocity versus time graph" ([15], p. 506). Students did not know which square they should include in the "area under the curve," so they counted all of the squares from under the curve all the way to the bottom line of the grid where the horizontal axis was labeled. That led to students' difficulties in distinguishing positive and negative areas, as well as associating them with displacement in the positive and negative direction, respectively.

More recently, Pollock et al. [16] investigated students' understanding of the physics and mathematics of process variables in $P-V$ diagrams in thermodynamics. On a question asking students to compare the work done by a gas taking two different paths on the $P-V$ diagram, they found that successful students were those who recognized that work was $\int P d V$ and that this integral equaled the area under the path.

These studies investigated students' application of the area under the curve in physics problems but were limited on the topics (i.e., the quantities that were calculated using the area under the curve). There have been no studies in physics education on how students related an integral and an area under the curve when there were several graphs provided. In our study, we investigate students' application of the area under the curve in physics problems covering a broader range of topics (e.g., work-energy, electric field, resistance, capacitance, electric current) and complexity, and also investigate how students relate an integral and an area under the curve when several graphs are provided. Specifically, we examine the following research questions:

RQ1: How did students in our study recognize the use of area under the curve in physics problems?

RQ2: How did students in our study understand what quantity was being accumulated when calculating the area under a curve?

RQ3: How did students in our study understand the relationship between a definite integral and the area under a curve?

In the next section, we will describe the format of our interviews as well as the rationale of the interview problems.

\section{METHODOLOGY}

\section{A. Interviews}

In the spring semester of 2009, 20 students were randomly selected from a pool of 102 volunteers enrolled in a first-semester calculus-based physics course (which we call Engineering Physics 1 or EP1) to participate in our study in mechanics. Most of these students were freshmen or sophomore engineering majors. Among these 20 students, there were 13 males and 7 females.

Each student was interviewed four times during the semester (interviews 1-4). The main reason that we chose 
this methodology was to gain deep insights into how students solve the interview problems, what difficulties they encounter, and what hints and scaffolding might help them overcome these difficulties. We call these interviews teaching-learning interviews [17]. This type of interview is different from the more commonly used clinical interview after Piaget [18,19]. While the focus of clinical interviews is to investigate the state of student knowledge-what people think-the main purpose of teaching-learning interviews is to investigate the process of knowledge construction-how people think and how they respond to prompts and hints from the interviewer. Since the purpose of our research was to investigate how students applied the area under the curve concept when solving physics problems, the teaching-learning interview was an appropriate methodology for our study.

Each interview occurred within two weeks after students had completed an exam in their physics course. The topics covered in the interviews were those that had been tested in the most recent exams. The topics were one-dimensional kinematics in interview 1, work and energy without friction in interview 2, work and energy with friction in interview 3, and rotational energy with friction in interview 4. In interview 1, students were asked to solve two problems: one from their recent exam and an isomorphic problem in which part of the information was given as a graph. In each of the interviews 2, 3, and 4, students solved three problems: an original problem, a graphical problem, and an algebraic problem. The original problem was selected from the most recent exam and was intended to prepare students with the physics concepts and principles used in the interview while the graphical and algebraic problems were modified versions of the original problem in which part of the information was provided as a graph or an algebraic expression of a function. In this paper, we focus our discussion on the graphical problem from interviews 2-4 because those problems involve calculating an integral using the area under the curve method.

In a related study, we investigated the interaction effect between graphical and algebraic representations [20]. For this purpose, approximately half of the participants in each interview were given the graphical problem before the algebraic problem (the G-A sequence) while the other half were given the algebraic problem before the graphical problem (the A-G sequence). We found that in both cases, better performance was observed in the problem that came later in the sequence, regardless of the representationgraphical or algebraic. This result indicated that students' performance on the second problem in the sequence was positively affected by the first problem. In this paper, we disregard this effect by only investigating those students who did the graphical problem first, i.e., followed the G-A sequence. The number of students following the G-A sequence in each of the interviews is presented in Table I.
TABLE I. Number of students following the G-A sequence in each interview.

\begin{tabular}{lc}
\hline \hline Interview & Number of students \\
\hline 2 & 11 \\
3 & 9 \\
4 & 9 \\
\hline \hline
\end{tabular}

In the fall semester of 2009, 15 students from among the spring 2009 interviewees, who were enrolled in a secondsemester calculus-based physics course (Engineering Physics 2 or EP2) at that time, agreed to continue participating in our study on electricity and magnetism. Among these 15 students, there were 9 males and 6 females. Each student went through another sequence of four interviews (interviews 5-8) during the semester. The format of these interviews was similar to that of the spring interviews, except that there were four to five problems in each interview. These problems included one problem with constant quantities and other problems with nonconstant quantities whose information was provided as graphs or algebraic expressions of functions. Each graph problem in the fall interviews contained three to four graphs of related quantities. All students were given the problems in the same order in all interviews. The topics of each interview were charge distribution and electric field in interview 5, resistance and capacitance in interview 6, current density and Ampere's law in interview 7, and $R L C$ circuit in interview 8 . We will only discuss the graphical problems from interviews 5, 6, and 7 because these problems involved matching a definite integral with an area under a curve.

All of the interviews in both semesters were conducted by the first author of this paper. Students were asked to think aloud as they solved the problems in our interviews. Verbal hints were given by the interviewer when students made a mistake or were on the wrong approach to the correct answer.

\section{B. Rationale of the interview problems}

Our interview problems were designed to investigate students' application of the area under the curve concept in calculating a physical quantity other than area. In the spring 2009 interviews, our problems were designed to answer the first two research questions:

RQ1: How did students in our study recognize the use of area under the curve in physics problems?

RQ2: How did students in our study understand what quantity was being accumulated when calculating the area under a curve?

The gun problem (interview 2) and the barrel problem (interview 3) were simple problems involving the area under the curve. Prior to our interviews, students had learned in the lecture that the work done by a force equaled the area under the curve of force versus displacement. In 
the gun and the barrel problems, students were provided with graphs of force versus linear displacement and they had to calculate the work done by a nonconstant force, so they only needed to recall the knowledge learned in the lecture. An interview episode in which the student was able to calculate the work from the graph of force in interview 2 is presented in Appendix A 1. For students who were not able to recall this knowledge, the interviewer would provide hints to help students recognize the use of the area under the curve in the problems. The set of hints that was used frequently to help students in the gun and the barrel problems included questions that led students to think about the multiplicative structure of the formula for the work and the area. Work was the product of force and displacement, and unit of work was the product of units of force and displacement. Then students were asked to think about how such a product could be obtained from the graph (i.e., multiplying the quantities on the vertical and horizontal axes, which essentially yielded the area). A typical interview episode in which the student was able to calculate the work after this set of hints was provided is presented in Appendix A 2. Another episode in which the student was unable to correctly respond to these hints and needed detailed guidance from the interviewer to calculate the work in interview 2 is presented in Appendix A 3.

The sphere problem (interview 4) was more complicated. This problem provided a graph of force versus angular displacement instead of linear displacement, so finding the area under the curve meant accumulating the product of force and angle, which did not yield the total work. Students had to convert angle to distance along the circular track by multiplying the angle by the radius of the track. Overall, this meant that students had to multiply the area under the curve by the radius of the track. So, this problem required an understanding of what quantity was being accumulated when computing the area under the curve. Therefore, it could help us determine whether students understood the relationship between work and area under the curve or just applied it as a rule. Once the student had recognized the use of the area under the curve in finding the work, the interviewer let the student calculate the area under the curve. If the student claimed that the area they obtained was the value of work, the interviewer would provide hints to help them recognize that it was in fact not the work yet and that they needed to multiply it with the radius of the track to calculate the correct value of work. The hints that were provided to the students asked them to focus on the unit of the area they calculated and compare this with the units of work. They were also provided hints that asked them to think about the relation between the angle and circumference of a full circle. Interview episodes of students who spontaneously recognized, recognized after hints, and recognized after detailed guidance that the area was not yet the value of work are presented in Appendices A 4-A 6, respectively.
As students proceeded through our interviews, they had become familiar with the use of area under the curve in physics problems, although as our results show they did not completely understand how to apply this knowledge in unfamiliar situations. Another important limitation of the interview problems chosen in spring 2009 was that, in all of these problems, students had one integral and were provided with one graph (i.e., the graph of the integrand versus the variable of integration); therefore, most students then knew that the integral equaled the area under the curve. If there had been more than one graph, we would have been able to investigate whether students knew under which curve to find the area. In order to choose from several graphs the graph corresponding to a predetermined integral, students had to understand the relation between integral and the area under the curve. So our problems in the fall 2009 interviews were designed to help us answer the third research question:

RQ3: How frequently did students in our study understand the relationship between a definite integral and area under a curve?

In each of these problems, students had to calculate a physical quantity (e.g., electric field, resistance, electric current) by evaluating a definite integral. Explicit expression of the integrand was neither given nor derived. Instead, students were provided with several graphs of related quantities. Students had to choose the graph on which the area under the curve equaled the integral at hand. This could help us determine whether students understood how a definite integral was related to area under a curve.

\section{Data analysis}

All interviews were video- and audiotaped and verbatim transcripts were created. Students' worksheets as well as interviewer's field notes were also collected. We first examined the field notes to identify interesting points in each interview and then referred to the student's worksheet and transcript for detail on what students wrote and said. We studied the transcripts of the interviews and looked for the answers to the following questions:

Did the student spontaneously recognize the use of the area under the curve in solving the problems?

If not, did the student recognize it after a few hints or after detailed guidance from the interviewer?

What hints were provided by the interviewer to help students recognize the use of the area under the curve?

We established an interrater reliability of $89 \%$ between two independent raters.

In the next section, we will present our findings from the interviews and discuss how these findings help us answer our research questions. We will use pseudonyms S1-S20 to identify the students. We used the same pseudonym to refer to the same individual in both semesters, i.e., student S13 in the spring 2009 interviews was the same student S13 in the fall 2009 interviews. 


\section{RESULTS}

\section{A. Calculating work from a graph of force versus position}

The graphical problems of interviews 2, 3, and 4 involved calculating the work done by a nonconstant force from a graph of force versus linear or angular position. Students learned from the lecture that the work done by a force equaled the area under the curve of force versus position. However, there was no homework or exam problem in which this knowledge was required, so students did not have a chance to practice finding work from a graph of force prior to our interviews.

We found that in interviews 2 and 3 , most of the students attempted to use the equation of work done by a constant force $W=F \cdot d$ or that of work done by the spring force $W=\frac{1}{2} k x^{2}$ to calculate work. Upon being asked to think of another strategy to find work, only a few students were able to recognize that they could find the area under the curve of force. Other students only recognized that the work equaled the area after hints or detailed guidance from the interviewer. In interview 4, students had become familiar with the task, so most of them spontaneously stated that work equaled area under the curve. However, the graph provided in interview 4 was a graph of force versus angular displacement instead of linear displacement, so the area under the curve did not yield work. To find the work done by frictional force in this problem, students had to find the area under the curve and multiply it by the radius of the circular track. This procedure was equivalent to calculating the integral $R \int_{0}^{\pi / 2} F(\theta) d \theta$ or $\int_{0}^{\pi R / 2} F(\theta) d s$, where $d s$ was an infinitesimal segment of length along the circular track.
We classified students' performance into three levels:

(i) getting the correct answer spontaneously, i.e., the student got the correct answer without any hints from the interviewer,

(ii) getting the correct answer after hints were provided by the interviewer, i.e., the student was able to use the area under the curve appropriately by following the hints provided by the interviewer,

(iii) getting the correct answer after detailed guidance from the interviewer, i.e., the student failed to follow the hints provided by the interviewer and needed to be told explicitly how to use the area under the curve.

The major difference between a student who got the correct answer after hints were provided and after detailed guidance was that the former student was able to respond correctly to the hints and obtained the correct answer by himself or herself, while the latter student was unable to respond correctly to the hints and needed to be told explicitly about how to arrive at the correct answer.

Examples of students who got the correct answer spontaneously, after a few hints, and after detailed guidance in interview 2 are presented in Appendices A 1-A 3 respectively. The problem in interview 3 was similar to that in interview 2, so we do not present examples for interview 3. Examples of students who recognize the need for the radius factor in the sphere problem (interview 4) spontaneously, after a few hints, and after detailed guidance are also presented in Appendices A 4-A 6 respectively.

The gun problem (Fig. 1).-This problem involved finding the work done by a spring force. There were two possible strategies for calculating the work done by the spring force in this problem. First, one could find the area under the curve of force versus displacement graph.

A $0.1 \mathrm{~kg}$ bullet is loaded into a gun (muzzle length $50 \mathrm{~cm}$ ) compressing a spring as shown. The gun is then tilted at an angle of $30^{\circ}$ and fired.

The only information you are given about the gun is that the barrel of the gun is frictionless and when the gun is held horizontally, the net force $F(N)$ exerted on a bullet by the spring as it leaves the fully compressed position varies as a function of its position $\mathrm{x}(\mathrm{m})$ in the barrel as shown in the graph below.

What is the muzzle velocity of the bullet as it leaves the gun, when the gun is fired at the $30^{\circ}$ angle as shown above?
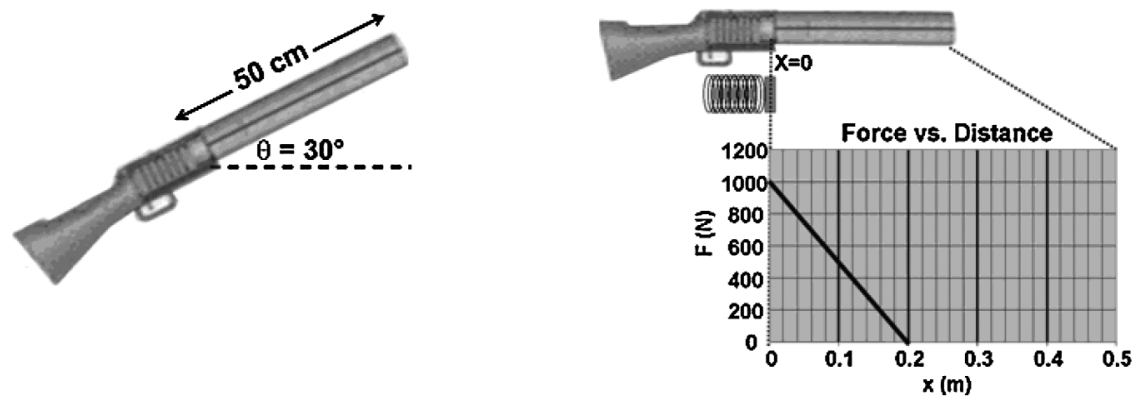

FIG. 1. The gun problem (interview 2). 
A $0.1 \mathrm{~kg}$ bullet is loaded into a gun compressing a spring which has spring constant $\mathrm{k}=6000 \mathrm{~N} / \mathrm{m}$. The gun is tilted vertically downward and the bullet is fired into a drum $5.0 \mathrm{~m}$ deep, filled with a liquid.

The barrel of the gun is frictionless. The resistance force provided by the liquid changes with depth as shown in the graph below. The bullet comes to rest at the bottom of the drum.

What is the spring compression $x$ ?
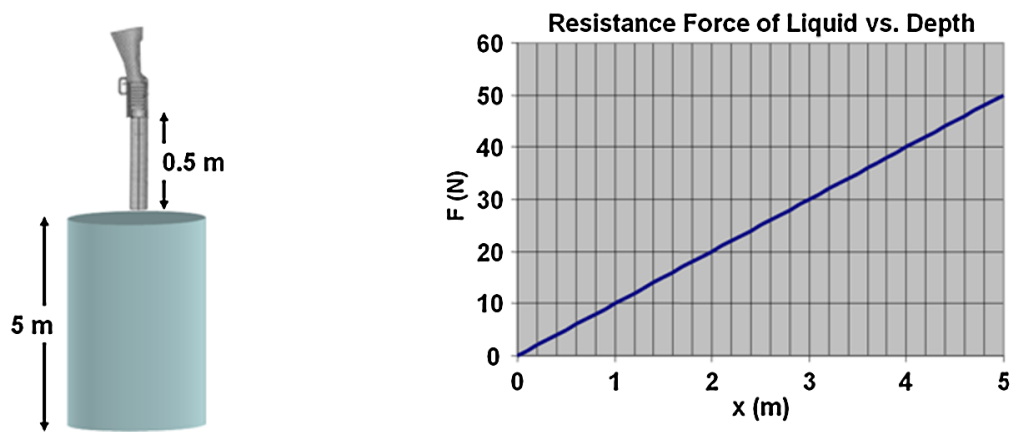

FIG. 2 (color online). The barrel problem (interview 3).

Second, one could find the spring constant $k$. Because of the linear dependence of spring force and displacement in this problem, the spring constant $k$ equaled the magnitude of the slope of the line. Then the work done by the spring force could be found from the equation $W=\frac{1}{2} k x^{2}$, where $x$ was the maximum spring compression.

Only one out of 11 students spontaneously stated that work equaled area under the curve of the force versus displacement graph and used the first strategy to calculate work. The other 10 students followed the second strategy and also obtained the correct value of work. When these students were asked to think of another strategy to find the work done by the spring force, six students could recognize that work equaled area under the curve of force after hints. The other four students stated that the area might have a physical meaning but were not able to recall what the meaning was until being explicitly told by the interviewer.

The barrel problem (Fig. 2).-This problem involved finding the work done by the resistance force of a liquid. This work might be found by either finding the area under the curve of force or treating the liquid as a spring whose spring constant was the slope of the line. Only three out of nine students spontaneously stated that work equaled area under the line. Three other students invoked the equation for work done by friction force on a horizontal floor $W=$ $F \cdot d=\mu m g d$ in which the coefficient of friction $\mu$ was the slope of the curve. Another student stated that the slope of the curve was the value of work. The remaining two students attempted to use the equation $W=F \cdot d$, where $F$ was the value of force at the maximum point on the graph. Of the six students who did not spontaneously calculate area under the curve, three recognized that work could be calculated using area under the curve after hints, while the other three were unable to recognize it until being told explicitly by the interviewer.
The sphere problem (Fig. 3).-This problem involved finding the work done by the rolling friction force on a circular track. This could be done by finding the area under the curve and multiplying this area by the radius of the track. Only one out of nine students spontaneously set up the correct calculation and got the correct value of the work. Five other students spontaneously stated that the area under the curve was the value of work. Of these five students, upon being told that the area itself was not the value of work, only two students recognized the need for the radius factor while the other three did not know what was missing and needed detailed guidance from the interviewer on both recognizing the use of area under the curve and the radius factor.

Table II summarizes the number of students (out of the total) who obtained the correct value of work using area under the curve without hints, with hints, and with detailed guidance.

From Table II, we see that only a few students (S9, S10, S15, S16) could spontaneously recognize the use of area under the curve in calculating work when the graph of force versus displacement was provided. Student S16 worked on the algebraic problem before the graphical problem in interview 4 so he was not included in the analysis of the sphere problem. In this problem, students S10 and S15 spontaneously stated that the work done by the rolling friction force was the area under the curve of force versus angle. One of them (S10) could recognize the need for the radius factor after being told that the area itself was not the value of work. The other student (S15) only obtained the correct value of work after detailed guidance from the interviewer. Student S9 was the only one who could calculate the correct value of work in the sphere problem without hints. However, he completed the graph problem after the algebraic problem in interviews 2 and 3, 
A sphere radius $r=1.0 \mathrm{~cm}$ and mass $m=2.0 \mathrm{~kg}$ is rolling at an initial speed $v_{i}$ of $5 \mathrm{~m} / \mathrm{s}$ along a track as shown. It hits a curved section (radius $R=1.0 \mathrm{~m}$ ) and is launched vertically at point A. The rolling friction on the straight section is negligible.

The magnitude of the rolling friction force acting on the sphere varies as angle $\theta$ as per the graph shown below.

What is the launch speed of the hoop as it leaves the curve at point A?
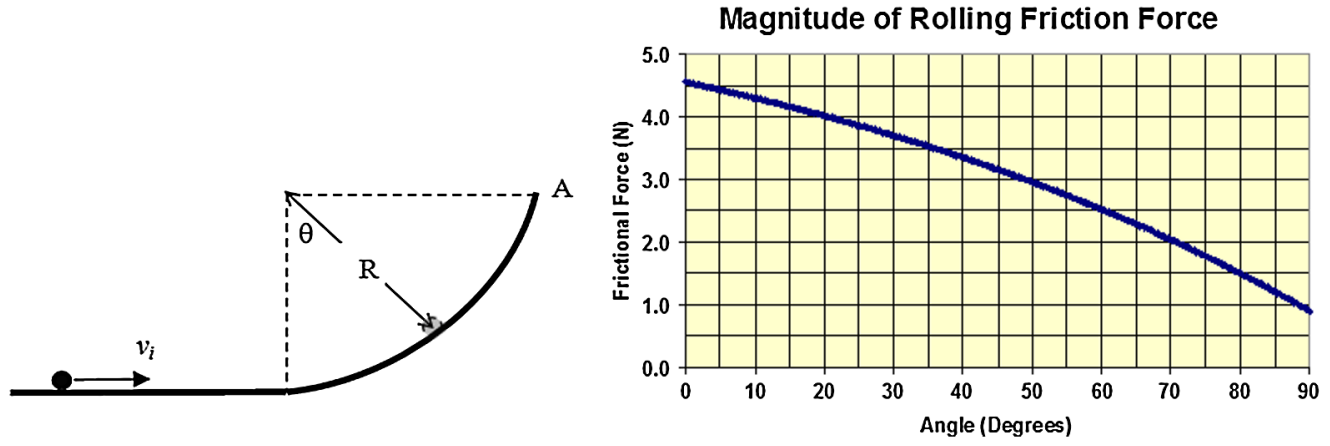

FIG. 3 (color online). The sphere problem (interview 4).

so he was not included in the analysis of the gun and the barrel problems. Therefore, we did not know whether or not he was able to recognize the use of area under the curve in those problems.

We answer our first two research questions as follows.

RQ1: How did students in our study recognize the use of area under the curve in physics problems?

The majority of students in our interviews did not spontaneously recognize the use of area under the curve in calculating work from the graph of force. There were two possible explanations: (i) students were not familiar with the method, and (ii) students held strong preferences for the algebraic method. The fact that more students were able to recognize that work equaled area under the curve as they progressed through the interviews suggests that students gained familiarity with the method. Some students, while talking with the interviewer after the interviews, stated that they had never seen a problem using area under the curve in their homework or on exams. On the other hand, students also expressed an inclination to an algebraic approach even when a graph was provided. They attempted to use prederived equations for work and just used the graph to collect data on the values of the spring constant or the coefficient of friction. Some students explicitly told the interviewer that they hated problems with graphs and preferred working with equations. These facts supported the second explanation.
RQ2: How did students in our study understand what quantity was being accumulated when calculating the area under a curve?

In the gun and barrel problems, the area under the curve itself was the value of work. So when a student recognized that work equaled area under the curve, we did not know whether he understood how work was accumulated when calculating the area or if he had just applied what he was taught in the lecture. There were four students in interview 2 who stated that the area had some meaning but they were unable to explain the meaning. In addition, there were also three students in interview 3 who stated that the slope of the line was the coefficient of friction. These were evidence that these students did not understand what quantity the slope and the area represented.

In the sphere problem, finding the area meant accumulating the product of force and angle, which was not work. Six out of nine students spontaneously stated that work equaled the area under the curve, but only one of them spontaneously recognized the need for the radius factor. This was further evidence that although students could invoke the statement that "work equals the area under the curve of force versus displacement," they might not understand what quantity was being accumulated when calculating such an area. Therefore, they failed to apply that knowledge in novel situations.

TABLE II. Students who obtained work using the area under the curve without hints, with hints, and with detailed guidance.

\begin{tabular}{lccc}
\hline \hline Problem & Correct without hints & Correct with hints & Correct with detailed guidance \\
\hline Gun & $1 / 11(\mathrm{~S} 16)$ & $6 / 11(\mathrm{~S} 1, \mathrm{~S} 2, \mathrm{~S} 4, \mathrm{~S} 8, \mathrm{~S} 19, \mathrm{~S} 14)$ & $4 / 11(\mathrm{~S} 3, \mathrm{~S} 7, \mathrm{~S} 11, \mathrm{~S} 12)$ \\
Barrel & $3 / 9(\mathrm{~S} 10, \mathrm{~S} 15, \mathrm{~S} 16)$ & $3 / 9(\mathrm{~S} 2, \mathrm{~S} 6, \mathrm{~S} 12)$ & $3 / 9(\mathrm{~S} 3, \mathrm{~S} 11, \mathrm{~S} 13)$ \\
Sphere & $1 / 9(\mathrm{~S} 9)$ & $2 / 9(\mathrm{~S} 6, \mathrm{~S} 10)$ & $6 / 9(\mathrm{~S} 5, \mathrm{~S} 17, \mathrm{~S} 18, \mathrm{~S} 13, \mathrm{~S} 15, \mathrm{~S} 20)$ \\
\hline \hline
\end{tabular}




\section{B. Matching a definite integral with an area under a curve}

The graphical problems of interviews 5, 6, and 7 involved evaluating definite integrals by calculating the areas under the curves. All 15 students (S1-S15) participating in these interviews solved the algebraic problems prior to the graphical problems. Each of the graphical problems provided three or four graphs describing the relation between the related quantities in the problem. Students had to select among these graphs the one in which the area under the curve was the value of the integral they encountered when solving the problem.

We found that most of the students preferred computing the integral algebraically to evaluating it graphically. Students attempted to find the expressions of the functions from the given graphs to plug into the integrals and computed them algebraically. Students considered evaluating the integrals using area under the curve only when the integral was too complicated to be computed algebraically or when students were unable to find the explicit expressions of the functions. About half of the students in each interview were able to select the appropriate graph to find area (i.e., the graph of the integrand), while others needed hints on this task. The hint provided to the students in this situation was to draw a graph of an arbitrary function $f(x)$ and have students label the axes of the graph such that the area under the curve from $a$ to $b$ equaled the integral $\int_{a}^{b} f(x) d x$. This exercise, which directed students' attention to the relation between the integrand and the function being plotted, helped most of the students recognize the correct graph to find the area. Interview episodes in which students were able to select the correct graph spontaneously and after hints are presented in Appendices A 7 and A 8 , respectively.

The arch problem (Fig. 4).- Students were given the graphs of " $\lambda(\theta)$ vs $\theta$," “ $\lambda(\theta) \sin \theta$ vs $\theta$," and " $\lambda(\theta) \cos \theta$ vs $\theta$, , and had to evaluate the integral $\int_{-\pi / 2}^{\pi / 2} \lambda(\theta) \cos \theta d \theta$. The value of this integral equaled the area under the curve of " $\lambda(\theta) \cos \theta$ vs $\theta$ " (i.e., the second graph in the problem statement) from $-\pi / 2$ to $\pi / 2$. One out of 15 students attempted to find the expression of $\lambda(\theta)$ to compute the integral algebraically. Four other students did not know what to do with the graphs. Upon being provided hints on the relation between a definite integral and an area under a curve, two of them were able to choose the correct graph to find area while the other two students needed further hints

You are standing at the center $O$ of the arch of radius $R$ in a stormy day. The arch is electrically insulated from the ground. There are negatively charged clouds over the arch. The charge distribution on the arch now depends on the angle $\theta$ as per the graphs shown.

Find the magnitude and direction of the electric field at your feet (i.e. at a point $O$ on the ground directly below the top of the arch).
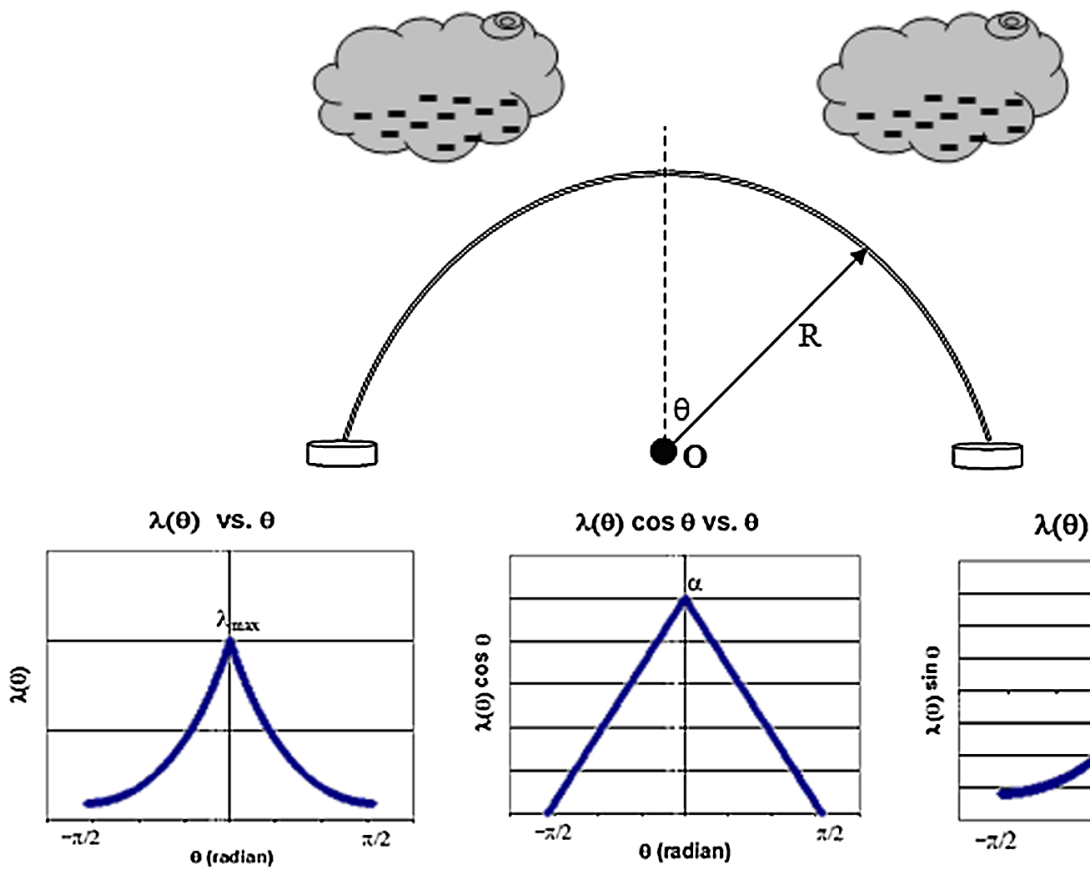

$\lambda(\theta) \cos \theta$ vs. $\theta$

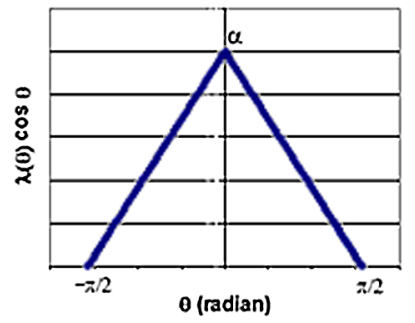

$\lambda(\theta) \sin \theta$ vs. $\theta$

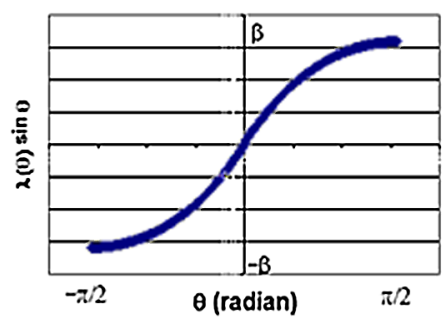

FIG. 4 (color online). The arch problem (interview 5). 
to recognize the correct graph. Out of the 10 students who spontaneously recognized the relation between integral and area under a curve, four students were able to choose the correct graph. The remaining six students initially chose an incorrect graph and needed hints to recognize the correct one. The errors these students made included: finding area under the curve of " $\lambda(\theta)$ vs $\theta$ " (S11, S14, and S15) because they were "integrating $\lambda(\theta)$," multiplying the area under the curve of " $\lambda(\theta)$ vs $\theta$ " by $\cos \theta$ (S6), choosing the graph of " $\lambda(\theta) \cos \theta$ vs $\theta$ " because "its area was easy to calculate" (S12), and relating the area with the antiderivative of the integrand (S13). The last error will be discussed as a misconception in Sec. IV C.

The conductor problem (Fig. 5).- Students were given the graphs of " $\rho(x)$ vs $x$." " $A(x)$ vs $x$," " $\rho(x) \cdot A(x)$ vs $x$," and " $\frac{\rho(x)}{A(x)}$ vs $x$," and had to evaluate the integral $R=$ $\int_{0}^{2} \frac{\rho(x)}{A(x)} d x$, where $\rho(x)$ and $A(x)$ were the resistivity and cross-sectional area of the conductor at position $x$. The value of this integral equaled the area under the curve of “ $\frac{\rho(x)}{A(x)} \mathrm{vs} x$ " (i.e., the fourth graph in the problem statement) from 0.0 to $2.0 \mathrm{~m}$. Three out of 15 students were able to choose the correct graph. Among the other 12 students, eight attempted to find the expression of $\rho(x)$ in order to compute the integral algebraically. The expression of the area function $A(x)$ had been derived in the algebraic problem which came before this problem. The integral obtained was too complicated to be computed algebraically, so these students considered evaluating the integral using area under the curve and were able to choose the correct graph. The remaining four students needed hints to recognize the correct graph to find area. The errors these students made could be attributed to students' misconceptions about basic properties of integration and the relation between an integral and the area under a curve. These misconceptions will be discussed in detail in Sec. IV C.

The current problem (Fig. 6).- - The equation for the current in this problem was $I=2 \pi \int_{0}^{2} j(r) r d r$. Students were given the graphs of " $j(r)$ vs $r$," " $r j(r)$ vs $r$," " $r^{2} j(r)$ vs $r$," and " $\frac{j(r)}{r}$ vs $r$." The value of the integral in the current equation equaled the area under the curve of $r j(r)$

A conductor has diameter decreasing from $\mathrm{D}$ to $\mathrm{d}$ over its length $\mathrm{L}=2.0 \mathrm{~m}$. The resistivity of this conductor along the $\mathrm{x}$ axis is $\rho(x)$ and its cross-sectional area is $A(x)$. The graphs of $\rho(x)$ vs. $x$, $A(x)$ vs. $x, \rho(x) . A(x)$ vs. $x$, and $\rho(x) / A(x)$ vs. $x$ are given. Find the resistance of this conductor.
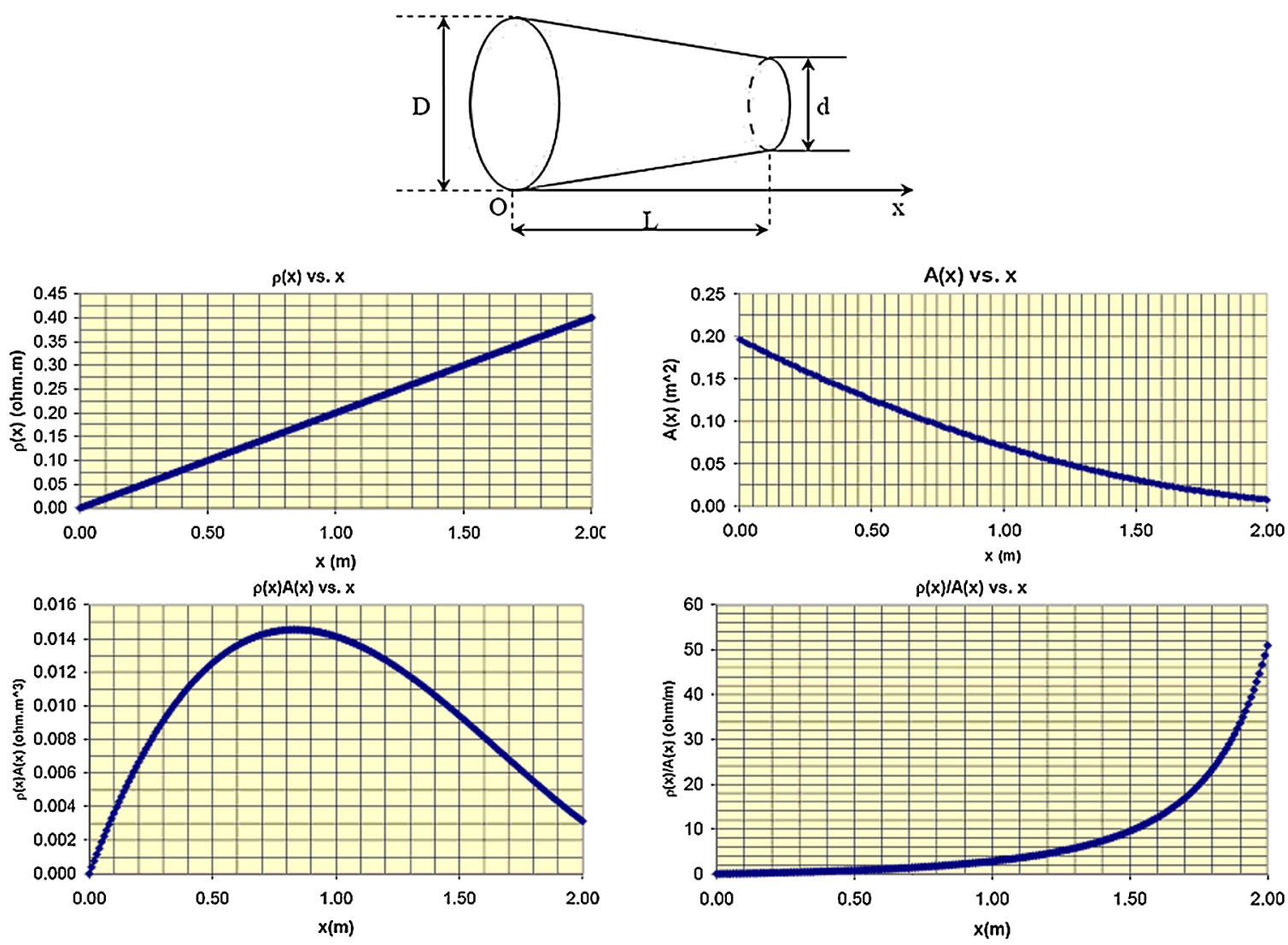

FIG. 5 (color online). The conductor problem (interview 6). 
A cylindrical wire of radius $\mathrm{R}=2 \mathrm{~cm}$ is carrying a current of density $j$ which depends on the distance $r$ from the center of the wire as per the graphs given. Find the magnitude of the magnetic field caused by the wire at a point $P$ on its surface.
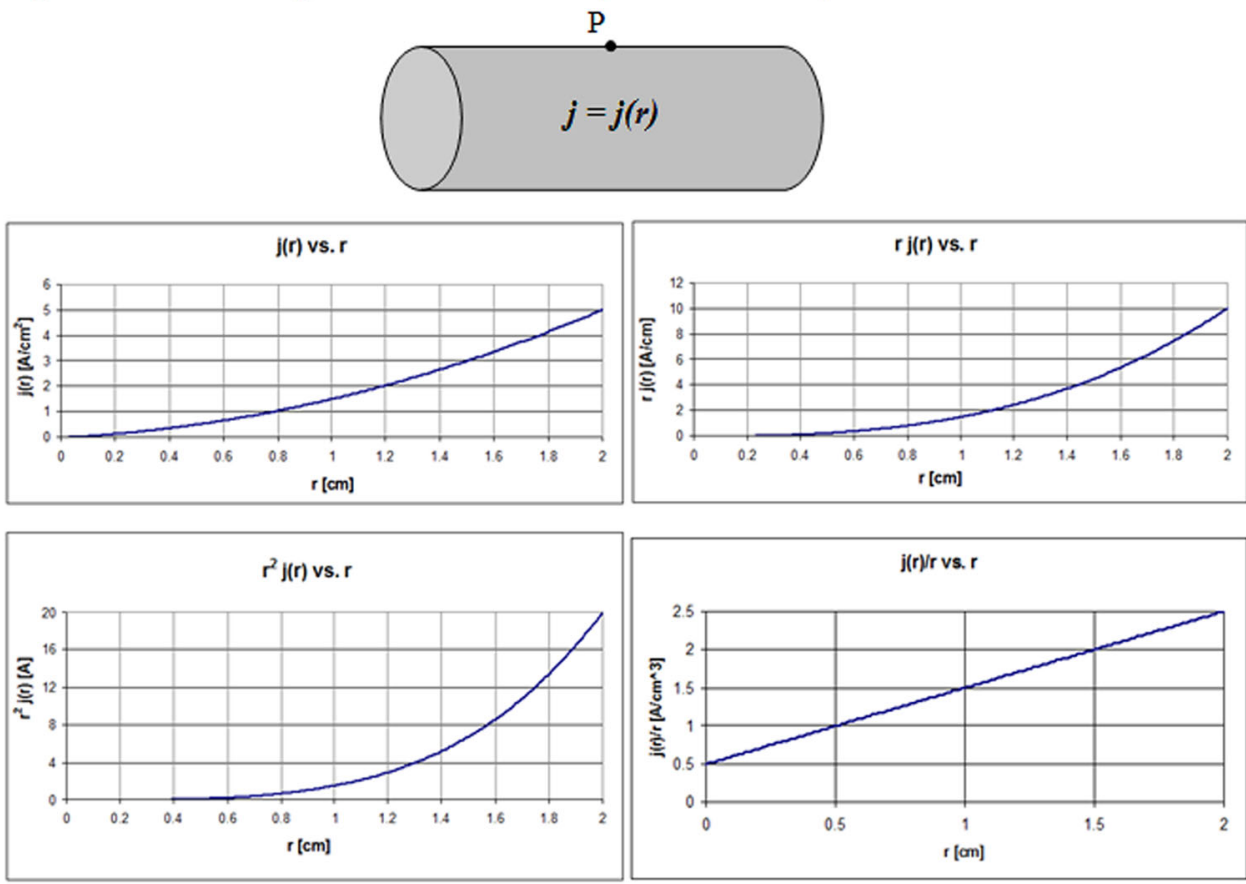

FIG. 6 (color online). The current problem (interview 7).

vs $r$ (i.e., the second graph in the problem statement) from 0 to $2 \mathrm{~cm}$. Nine out of 15 students were able to choose the correct graph. Four other students chose the " $j(r)$ vs $r$ " graph for the reason that the current density $j(r)$ was being integrated. The remaining two students chose the " $\frac{j(r)}{r}$ vs $r$ " graph because its area was straightforward to calculate.

In summary, almost all of the students indicated knowledge that an integral equaled the area under a curve. However, when provided with several graphs, students had difficulties identifying the graph under which the area was the value of a certain integral. There were four common errors that students made in selecting the graph:

(i) relating only one part of the integrand with the function being plotted [e.g., equating $\int_{-\pi / 2}^{\pi / 2} \lambda(\theta) \cos \theta d \theta$ with the area under the curve of " $\lambda(\theta)$ vs $\theta$ " or $\int_{0}^{2} j(r) r d r$ with area under the curve of " $j(r)$ vs $r$ "];

(ii) relating the area with the integrand (e.g., equating the area under the curve of " $\rho(x)$ vs $x$ " with the value of the function $\rho(x)$ in the integral);

(iii) identifying the graph to find the area based on the simplicity of the area calculation (e.g., choosing a graph because the area calculation was straightforward);

(iv) applying incorrect properties of integration (e.g., equating the integral of a quotient with the quotient of integrals).

In Sec. IV C, we will discuss students' misconceptions about integration and the area under a curve.

\section{Students' misconceptions about the integral and the area under a curve}

Our interviews also revealed some students' misconceptions about basic properties of integrals and the relationship between the integrals and the area under a curve. These misconceptions were the integral equals the area under the curve of the antiderivative of the integrand, integral of a product or quotient equals sum or quotient of integrals, and integrand equals area under the curve. We will discuss each of these misconceptions below.

Area under a curve equals the antiderivative of the integrand - In the arch problem (interview 5), the integral was $\int_{-\pi / 2}^{\pi / 2} \lambda(\theta) \cos \theta d \theta$, which equaled the area under the curve of " $\lambda(\theta) \cos \theta$ vs $\theta$ " from $-\pi / 2$ to $\pi / 2$. Student S13 chose the graph of " $\lambda(\theta) \sin \theta$ vs $\theta$ "] because she integrated $\cos \theta$ before finding the area. The transcript of this interview episode is presented in Appendix A 9.

The area under a curve equals the integrand.-In the conductor problem (interview 6), the integral was $\int_{0}^{2} \frac{\rho(x)}{A(x)} d x$, which equaled the area under the curve of $\frac{\rho(x)}{A(x)}$ vs $x$ " from 0.0 to $2.0 \mathrm{~m}$. Student $\mathrm{S} 8$ calculated the areas under the curves of " $\rho(x)$ vs $x$ " and " $A(x)$ vs $x$ " and plugged those areas into $\rho(x)$ and $A(x)$ in the integral. Similarly, in the current problem (interview 7), student S3 calculated the area under the curve of " $j(x)$ vs $r$ " and plugged that area into $j(r)$ in the integral $\int_{0}^{2} j(r) r d r$. These errors suggested that these students 
perceived the area under a curve as the value of the integrand rather than the value of the integral. The transcript of the interview episode in which this type of misconception occurred is presented in Appendix A 10.

Integral of a product or quotient equaled a sum or quotient of integrals.-In the conductor problem (interview 6), student $\mathrm{S} 1$ found the explicit expression of $\rho(x)$ from the " $\rho(x)$ vs $x$ " graph and calculated the integral using the equation $\int_{0}^{2} \frac{\rho(x)}{A(x)} d x=\int_{0}^{2} \rho(x) d x+\int_{0}^{2} \frac{d x}{A(x)}$. Students S6 and S8 attempted to use the equation $\int_{0}^{2} \frac{\rho(x)}{A(x)} d x=\frac{\int_{0}^{2} \rho(x) d x}{\int_{0}^{2} A(x) d x}$ and calculated the quotient of the areas under the curves of " $\rho(x)$ vs $x$ " and " $A(x)$ vs $x$." The transcript of the interview episode in which this type of misconception occurred is presented in Appendix A 11.

In summary, we found evidence that students might not completely understand the concept that "the integral equals the area under the curve" although they might be able to invoke it during problem solving. We also found evidence that some students held misconceptions about basic properties of integrals.

We answer our last research question-RQ3: How did students understand the relationship between a definite integral and area under a curve?

Almost all of the students indicated knowledge of "the integral equaled the area under the curve," but only half of them (four students in interview 5, eight in interview 6, and nine in interview 7) were able to select the graph corresponding to a predetermined integral when several graphs were present. The errors other students made - choosing a graph based on part of the integrand or on the simplicity of the area calculation-indicated that these students did not completely understand the relationship between a definite integral and area under a curve.

\section{DISCUSSION}

In this study, we found that the majority of the students did not spontaneously invoke the area under the curve concept during physics problem solving. This might be attributed to students' unfamiliarity with the graphical methods as well as their strong inclination toward algebraic methods in solving physics problems. Even when students invoked the area under the curve concept in a physics problem, there was evidence that they might not understand what physical quantity the area represented. We also found that when provided with several graphs, many students were unable to choose the graph on which the area under the curve equaled a predetermined integral, even though they could state that the integral equaled the area under the curve.

We will now discuss how our findings are consistent with other studies in mathematics and physics education research on students' use of the area under the curve concept.
Students' difficulties with the area under the curve concept in the physics context of our study are similar to those previously found in mathematics context. We found that most of the students used area under the curve to find work from a graph of force versus displacement, but they might not understand why the work was equal to the area, so they failed to recognize that the area under the curve in the sphere problem was not yet the value of work. This is similar to what Artigue concluded in his study [5]: most students could perform routine procedures of finding area under the curve but rarely could they explain why these procedures were necessary.

Thompson and Silverman [13] suggested that for students to perceive the area under the curve as representing a quantity other than area (in our case it was work), students must be able to see the integration process as an accumulation of the incremental bits that were formed multiplicatively. The hints we provided to help students recognize the use of the area under the curve concept in our interviews aimed at this goal. We asked students questions that directed their attention to the fact that the total work was the accumulation of the product of force and distance over small increments, which was essentially the area under the curve on the graph of force versus linear displacement.

Sealey [14] concluded that the area under the curve method could be a powerful tool to evaluate a definite integral only when students understood the structure of the definite integral. Our study showed the extent to which students struggled with choosing an area that equaled a definite integral when they did not view the integral as having two components: the integrand and the infinitesimal term $d x$ or $d r$. About half of the students in our interviews chose the incorrect graph because their choice was based on the wrong clues (i.e., based on part of the integrand, the antiderivative of the integrand, or the ease of finding the area). The hints that asked students to label a graph of an arbitrary function $f(x)$ such that the area under the curve equaled the integral $\int_{a}^{b} f(x) d x$ directed students' attention to the two components of an integral and helped them recognize that the integrand was the clue for choosing the correct graph.

McDermott et al. [15] studied how students used area under the curve in kinematics. Our study investigated students' use of area under the curve in many other topics of introductory physics. We did not have any problems involving negative area as in the study by McDermott et al., but we had problems with more than one graph from which we could investigate how students related a definite integral with an area under a curve.

\section{LIMITATIONS AND FUTURE WORK}

The research methodology used in this study was the individual interview. This method had an advantage in that 
it allowed us to gain insight into how individual students interacted with the concept of an integral as area under the curve. It also allowed us to interview the same students several times during two semesters, and therefore, we could track the development of the students through both of the courses. In spite of the advantages afforded by individual interviews, the method limited the number of students participating in the study, and hence, limited the generalizability of the results.

Our interview problems involved several physics quantities that could be calculated using area under the curve. However, there was no problem involving negative areas or areas that had the lower bound other than the $f(x)=0$ axis (i.e., the $x$ axis). By "area under the curve" we usually mean the area bounded by the curve and the $f(x)=0$ axis. There are problems in which the "area under the curve" is bounded by the curve and the $f(x)=-2$ line for instance. Investigating whether students know that "integral equals area under the curve, but above what?" will be an interesting study following the study presented in this paper.

Based on our interview findings, we plan to develop tutorial materials to help students understand the "integral equals area under the curve" relationship and implement them for all of the students in both EP1 and EP2 courses (usually around $200+$ students each) in future semesters when the courses are offered to test the effects of those materials in helping students learn to use the area under the curve method in physics problem solving.

\section{ACKNOWLEDGMENTS}

We gratefully acknowledge the contributions of Dr. Elizabeth Gire, currently at University of Memphis, for her participations in the discussions on the design of the interview questions for fall 2009. This work is supported in part by U.S. National Science Foundation Grant No. 0816207.

\section{APPENDIX A}

\section{An interview episode in which a student spontaneously recognized the use of the area under the curve in interview 2}

Interviewer: So what are you trying to calculate?

Student: Spring constant based on the graph ... which isn't necessary actually because $W=F \cdot d \ldots$ because I can just have the work on the ball so I'll just need to integrate the graph. Since it's a triangle I would say $\frac{1}{2} b$. $h=W=100 J$ is work done by the spring.

In this episode, the student recognized that he could find the work done by the spring force by saying that he could "integrate the graph" and his calculation indicates that he meant finding the area under the curve of force versus distance.

\section{An interview episode in which a student recognized the use of the area under the curve after a few hints in interview 2}

Interviewer: So you found $x$ and $F$ to find $k$ to use in potential energy. But can you think of a way to find potential energy without knowing $k$ and $x$ ?

Student:... I'm not sure, that's the only equation I know for it.

Interviewer: What is the equation for work?

Student: $W=F \cdot d$

Interviewer: How are force and distance given on the graph?

Student: They are plotted on the graph.

Interviewer: Force is plotted on the vertical axis and distance on the horizontal axis.

Student: Yeah.

Interviewer: So work is the product of these two quantities, which means it is the product of the vertical and horizontal values. What do you get when you multiply the horizontal and vertical dimensions of a graph?

Student: I'll get the area of the graph.

Interviewer: Right, so you know how to find work now.

Student: Yeah ... area under the curve.

In this episode, the student started out knowing only one method for calculating the work: using the formula $W=\frac{1}{2} k x^{2}$. Being prompted that the work was the product of force and distance, which were the two dimensions of the given graph, the student was able to recognize that such a product was the area of the graph, and therefore, the work was the area bounded by the curve of force.

\section{An interview episode in which a student recognized the use of the area under the curve after detailed guidance in interview 2}

Interviewer: You found $k$ by finding $F$ and $x$ but there is another way without knowing $x$ and knowing $F$. Can you think of any other method?

Student: I guess the graph tells you.

Interviewer: Ok, how will you do that?

Student: Well, at $x=0.2$ then potential energy is 1000 .

Interviewer: Not potential energy. The only thing that tells you is that the force is $1000 \mathrm{~N}$ at $x=0.2$. Potential energy is equal to the work done by the spring.

Student: Which is $F \cdot d$.

Interviewer: Yes, how do you get the force and distance?

Student: This graph tells you the force at different distance ... I don't know.

Interviewer: Force is the vertical axis on the graph, and distance is the horizontal axis. So how can you find $F \cdot d$ on the graph?

Student: I have no idea.

Interviewer: Okay, multiplying the vertical and the horizontal axes of the graphs, you'll get its area. So in this case work is the area under the curve.

Student: Oh yes. 
In this episode, the interviewer provided similar hints as in the episode presented in Appendix A 2. However, the student still could not recognize that the work equaled the area under the curve until being told explicitly by the interviewer.

\section{An interview episode in which a student spontaneously recognized the need for the unit conversion in interview 4}

The following episode started after the student had calculated the area under the curve and got 273 area units.

Student: This is my area under the curve, which is work done by the force ... Oh wait, the unit is not right.

Interviewer: What is the unit of that number?

Student: It's Newton times degree now, but I need Joule.

Interviewer: So what should you do then?

Student: I think I can change it. [drew a unit conversion table and converted the unit correctly and got $4.76 \mathrm{~J}$ ]

Interviewer: Yeah, that's right.

In this episode, the student first claimed that the area under the curve was the work done by the force. But then he spontaneously recognized that the unit was not right, and was able to convert the unit by himself.

\section{An interview episode in which a student recognized the need for the radius factor after hints in interview 4}

The following episode started after the student had calculated the area under the curve and got 270 area units.

Interviewer: What is the unit of that number?

Student: Umm ... Newton time degree.

Interviewer: How do you know?

Student: ... because you're finding the area multiplying this [horizontal axis] times this [vertical axis] which is Newton time degree.

Interviewer: What do you want to calculate?

Student: Work.

Interviewer: What is the unit of work?

Student: It's Joule, isn't it?

Interviewer: Yes, but what is a Joule?

Student: Joule is ... Newton ... per ... meter squared.

Interviewer: What is the equation for the work done by a force?

Student: Work is force time distance.

Interviewer: Right, so what is the unit of work then?

Student: Newton time meter.

Interviewer: But here you have Newton times degree and you have to convert that to Newton times meter.

Student: How do I find degrees from meter? I've never even heard of going from degree to meters...

Interviewer: How many degrees are there in a full circle? Student: 360.

Interviewer: How many meters are there in a full circle? I mean the circumference.

Student: $2 \pi R \ldots$ So I have $\ldots \frac{2 \pi R \text { meters }}{360 \text { degrees }}$
Interviewer: Right, so now you can convert the unit.

Student: [did the unit conversion and got the correct value of work]

In this episode, the student did not spontaneously recognize the need for a unit conversion until being prompted that the unit of the area under the curve was not the unit of work. The student then did not know how she could convert the unit because she "never even heard of going from degrees to meters." She was, however, able to respond correctly to the hints provided by the interviewer and was able to set up the unit conversion factor.

\section{An interview episode in which a student recognized the need for the radius factor after detailed guidance in interview 4}

The following episode started after the student had calculated the area under the curve and got 267.5 area units.

Interviewer: That is right, but what is the unit?

Student: Newton ... per meter?

Interviewer: You found the area by multiplying the $x$-values by the $y$-values so what is the unit of the area?

Student:... per degree?

Interviewer: The $y$-value is in Newton and the $x$-value is in degree and you multiply them so what is the unit?

Student: Newton time degree.

Interviewer: Yes. Newton time degree.

Student: Oh and that equals the work, right?

Interviewer: What is the unit of work?

Student: Umm ... Newton ... per meter?

Interviewer: Newton time meter. So how can you convert the unit?

Student: I don't know because it's not in radians and if it was in radians then I would multiply it by $2 \pi$ or something like that.

Interviewer: Okay, let's consider the full circle how many degrees?

Student: 360.

Interviewer: How many meters are there in a full circle? I mean the circumference.

Student: $2 \pi$ or something ...

Interviewer: $2 \pi R$. So 360 degrees are corresponding to $2 \pi R$ meters, so what is the unit conversion factor now?

Student: Umm ... I'm confused. Why we are talking about a full circle but we just have a part of a circle?

Interviewer: That is just an example for you to see how a degree is related to a meter.

Student: $R=1 \mathrm{~m}$ so the circumference is $2 \pi$ meters, not $1 \mathrm{~m}$.

Interviewer: What I mean is that you can make a fraction which equals 1 and has the unit of meter per degree.

Student: Umm ... I don't know how I can get 1 from these. 
Interviewer: Okay, 360 degrees are corresponding to $2 \pi R$ meters, so the fraction is $\frac{2 \pi R \text { meters }}{360 \text { degrees }}$. Now you can do the unit conversion.

Student: I don't know how to convert unit though.

Interviewer: Alright, the unit conversion is $(267.5 \mathrm{~N} \times$ degrees) $\frac{2 \pi R \text { meters }}{360 \text { degrees }}$ and you'll get a number in Newton time meter, which is Joule. And that's the value of work.

Student: Okay.

The student in this episode claimed that the area under the curve was the value of work. He was not able to state the correct unit of it upon being asked. He seemed to be completely lost when the interviewer attempted to provide hints on unit conversion, and needed to be told explicitly how to convert the unit.

\section{An interview episode in which a student spontaneously selected the correct graph to find the area in interview 5}

The following episode started after the student had set up the correct integral for the electric field $E=\frac{1}{4 \pi \varepsilon_{0} R} \times$ $\int_{-\pi / 2}^{\pi / 2} \lambda(\theta) \cos \theta d \theta$.

Student: I have this integral and I have the graphs so I have to use the area under the curve then.

Interviewer: Which curve do you use?

Student: This one. [points at the graph of " $\lambda(\theta) \cos \theta$ vs $\left.\theta^{\prime \prime}\right]$

Interviewer: How do you know?

Student: Since I'm integrating $\lambda(\theta) \cos \theta$.

Interviewer: Yes, right.

In this episode, the student was able to recognize that he needed to use the graph of " $\lambda(\theta) \cos \theta$ vs $\theta$ " and his reasoning indicates that he chose the curve based on the integrand.

\section{An interview episode in which a student selected the correct graph to find the area after hints in interview 5}

The following episode started after the student had set up the correct integral for the electric field $E=\frac{1}{4 \pi \varepsilon_{0} R} \times$ $\int_{-\pi / 2}^{\pi / 2} \lambda(\theta) \cos \theta d \theta$.

Interviewer: How can you evaluate this integral?

Student: I can find the area under the curve.

Interviewer: Which curve do you use?

Student: I'm integrating $\lambda$ and this graph [points at the graph of " $\lambda(\theta)$ vs $\theta$ "] tells me about $\lambda$.

Interviewer: Other graphs also have $\lambda$.

Student: So ... I don't know which one then.

Interviewer: Okay, now if I have a curve [draw an arbitrary curve] and I tell you that the area under this curve from $x=a$ to $x=b$ equals the integral $\int_{a}^{b} f(x) d x$, can you label the axes of the graph?

Student: This is $f(x)$ [labels the vertical axis] and this is $x$ [labels the horizontal axis].
Interviewer: Right, but how did you know?

Student: Because the integral equals the area under the curve.

Interviewer: So do you know which curve to find the area now?

Student: Well ... here I have $\lambda(\theta)$ instead of $f(x) \ldots$ so I want to say this graph [points at the graph of " $\lambda(\theta)$ vs $\theta$ "] but you told me it's not right.

Interviewer: What is the integrand in this integral?

Student: Oh ... I have to integrate $\cos \theta$ too. So I would say this graph. [points at the graph of " $\lambda(\theta) \cos \theta$ vs $\theta$ "]

Interviewer: You get it now.

The student in this episode first chose the graph of " $\lambda(\theta)$ vs $\theta$ " because "it tells me about $\lambda$." With the hints provided by the interviewer, he was able to recognize that he needed to use the graph of " $\lambda(\theta) \cos \theta$ vs $\theta$ ".

\section{An interview episode in which a student selected the graph to find the area based on the antiderivative of the integrand in interview 5}

The following episode started after the student had set up the correct integral for the electric field $E=\frac{1}{4 \pi \varepsilon_{0} R} \times$ $\int_{-\pi / 2}^{\pi / 2} \lambda(\theta) \cos \theta d \theta$.

Interviewer: Okay, so now you have graphs ...

Student: Yeah, I understand that I have to use these graphs, I just don't know how.

Interviewer: And you have your integral. So what is the relation between an integral and a graph?

Student: It's the area underneath the curve.

Interviewer: Uh huh, area under the curve. So which graph do you use to find the area?

Student: I'm hoping this one. [points at the graph of " $\lambda(\theta)$ vs. $\theta$ "]

Interviewer: Yes, you hope. But you should have a reason.

Student: No ... It's this one. [points at the graph of " $\lambda(\theta)$ vs. $\theta$ "]

Interviewer: How do you know you should use that graph?

Student: Um, because if I take the integral of cosine it's going to be sine so I need the area under this.

This student was able to recognize that the integral equals the "area underneath the curve" when provided hints on the relation between integral and graph. However, she was not sure which area was corresponding to the integral. After picking a graph with the "hope" that it would be the correct one, she was more thoughtful in her second attempt. Her explanation that the integral of cosine was sine indicated that she chose the graph based on the result of integrating the cosine in the integrand. This evidence suggested that she did not understand the relation "the integral equals the area underneath the curve" although she could invoke it when solving the problem. 


\section{An interview episode in which a student found the area under the curve and used it as the integrand to find the area in interview 6}

The following episode started after the student had set up the integral for the resistance $R=\int_{0}^{2} \frac{\rho(x)}{A(x)} d x$.

Student: I need to find ... do the area under this curve... [points at the graph of " $\rho(x)$ vs $x$ "] . . and this curve ... [points at the graph of " $A(x)$ vs $x$ "]

Interviewer: How do you know?

Student: 'Cause I need $\rho(x)$ and $A(x)$ in the integral.

Interviewer: So what do you plan to do?

Student: I need the areas to plug in the integral.

Interviewer: No, the area itself is already the integral.

Student: Oh ... okay, so I will use this graph then. [points at the graph of “ $\frac{\rho(x)}{A(x)}$ vs $x$ ”]

Interviewer: Let's do that.

In this episode, the student attempted to find the areas under the curves of " $\rho(x)$ vs $x$ " and " $A(x)$ vs $x$ " to plug in the integral. After being told that the area equaled the whole integral, not just the integrand, he was able to choose the correct graph.

\section{An interview episode in which a student selected the graph to find the area based on incorrect property of the integral in interview 6}

The following episode started after the student had set up the integral for the resistance $R=\int_{0}^{2} \frac{\rho(x)}{A(x)} d x$.
Student: Now I have to find the area underneath the curve.

Interviewer: Which curve do you use?

Student: You actually have two functions here, so I'll need two areas.

Interviewer: What are the areas?

Student: Area under this one and this one. [points at the graph of " $\rho(x)$ vs $x$ " and " $A(x)$ vs $x$ "]

Interviewer: What will you do with those areas?

Student: Since it's a division here so I have to divide the areas.

Interviewer: You actually can't do that because you have $\int_{0}^{2} \frac{\rho(x)}{A(x)} d x$ and what you did was to find $\frac{\int_{0}^{2} \rho(x) d x}{\int_{0}^{2} A(x) d x}$. They are not equal.

Student: Umm ... so then I would say this graph. [points at the graph of “ $\frac{\rho(x)}{A(x)}$ vs $x$ "]

Interviewer: Yeah, that's right.

The student in this episode noticed that he had two functions so he needed two areas. He also attempted to divide the areas, and recognized the correct graph after being told that the integral of a quotient was not the quotient of integrals. These indicate that he was relating the area under the curve with the individual function, not the whole integral, and was holding a misconception about the property of the integral.
[1] D. C. Meredith and K. A. Marrongelle, How students use mathematical resources in an electrostatics context, Am. J. Phys. 76, 570 (2008).

[2] L. Cui, A. Bennett, P. Fletcher, and N. S. Rebello, Transfer of learning from college calculus to physics courses, Proceedings of the Annual Meeting of the National Association for Research in Science Teaching, 2006 (unpublished).

[3] F. R. Yeatts and J. R. Hundhausen, Calculus and physics: Challenges at the interface, Am. J. Phys. 60, 716 (1992).

[4] A. Orton, Students' understanding of integration, Educ. Stud. Math. 14, 1 (1983).

[5] M. Artigue, Analysis, in Advanced Mathematical Thinking, edited by D. Tall (Kluwer, Boston, 1991), pp. 167-198.

[6] J. Ferrini-Mundy and K. Graham, Research in calculus learning: Understanding of limits, derivatives, and integrals, in Research Issues in Undergraduate Mathematics Learning: Preliminary Analyses and Results, edited by J. Kaput and E. Dubinsky (Mathematical Association of America, Washington, DC, 1994).

[7] D. O. Tall and S. Vinner, Concept image and concept definition in mathematics, with particular reference to limits and continuity, Educ. Stud. Math. 12, 151 (1981).
[8] J. Bezuidenhout and A. Olivier, Students' conceptions of the integral, in Proceedings of the 24th Conference of the International Group for the Psychology of Mathematics Education, Hiroshima, Japan, 2000 (unpublished).

[9] S. Rasslan and D. Tall, Definitions and images for the definite integral concept, in Proceedings of the 26th Conference on Psychology of Mathematics Education, Norwich, 2002 (unpublished).

[10] T. Grundmeier, J. Hansen, and E. Sousa, An exploration of definition and procedural fluency in integral calculus, Primus 16, 178 (2006).

[11] N. Mahir, Conceptual and procedural performance of undergraduate students in integration, Int. J. Math. Educ. Sci. Technol. 40, 201 (2009).

[12] K. K. Chapell and K. Killpatrick, Effects of concept-based instruction on students' conceptual understanding and procedural knowledge of calculus, Primus 13, 17 (2003).

[13] P. W. Thompson and J. Silverman, The concept of accumulation in calculus, in Making the Connection: Research and Teaching in Undergraduate Mathematics, edited by M.P. Carlson and C. Rasmussen (Mathematical Association of America, Washington, DC, 2008), pp. 43-52. 
[14] V. Sealey, Definite integrals, Riemann sums, and area under a curve: What is necessary and sufficient?, in Proceedings of the 28th Annual Meeting of the North American Chapter of the International Group for the Psychology of Mathematics Education, Merida, Mexico, 2006 (unpublished).

[15] L.C. McDermott, M.L. Rosenquist, and E.H. van Zee, Student difficulties in connecting graphs and physics: Examples from kinematics, Am. J. Phys. 55, 503 (1987).

[16] E. Pollock, J. Thompson, and D. Mountcastle, Students' understanding of the physics and mathematics of process variables in $P-V$ diagram, in Proceedings of the Physics Education Research Conference, 2007 (unpublished).
[17] P. V. Engelhardt, E. G. Corpuz, D. J. Ozimek, and N.S. Rebello, The Teaching Experiment-What it is and what it isn't, in Proceedings of the Physics Education Research Conference, Madison, WI, 2003 (unpublished).

[18] J. Piaget, The Child's Conception of the World (Harcourt, Brace, New York, 1929).

[19] J. Piaget, The Child's Conception of Physical Causality (Harcourt, Brace, New York, 1930).

[20] D. Nguyen and N.S. Rebello, Students' difficulties in transfer of problem solving across representations, in Proceedings of the 2009 Physics Education Research Conference, Ann Arbor, MI, edited by M. Sabella, C. Henderson, and C. Singh (AIP, New York, 2009), Vol. 1179. 